\title{
THE USE OF SPECTRAL METHOD FOR FATIGUE LIFE ASSESSMENT FOR NON-GAUSSIAN RANDOM LOADS
}

\author{
Adam NIESŁONY*, Michał BÖHM*, Tadeusz ŁAGODA*, Filippo CIANETTI* \\ *Faculty of Mechanical Engineering, Department of Mechanics and Machine Design, Opole University of Technology, \\ ul. Mikołajczyka 5, 45-271 Opole, Poland \\ *Department of Engineering, University of Perugia, via G. Duranti, 93, Perugia, 06125 Italy \\ a.nieslony@po.opole.pl, m.bohm@po.opole.pl, t.lagoda@po.opole.pl, filippo.cianetti@unipg.it
}

received 4 May 2015, revised 10 May 2016, accepted 12 May 2016

\begin{abstract}
The well-known problem with the fatigue lifetime assessment of non-Gaussian loading signals with the use of spectral method has been presented in the paper. A correction factors that transform the non-Gaussian signal into an equivalent Gaussian signal proposed by Bracessi et al. (2009) has been used for the purpose of lifetime calculations together with Palmgren-Miner Hypothesis. The calculations have been performed for the 10HNAP steel under random non-Gaussian load with four dominating frequencies. The signal has been generated on the test stand SHM250 for random tension-compression tests. The results with zero and non-zero mean stresses have been used to calculate the fatigue life with the frequency domain method based on Dirlik's model and with a time domain method with the use of the rainflow cycle counting algorithm. The obtained calculation results have been compared with experimental results.
\end{abstract}

Key words: Spectral Method, Fatigue Life, Random Loading, Non-Gaussian

\section{INTRODUCTION}

Fatigue life assessment is an important issue nowadays, and is strongly related with the reliability of components. It gains importance if we are dealing with random loading conditions or vibrations (Benasciutti et al. 2006, 2010, 2013). Some scientists have noticed that most stochastic natural forces as e.g. the wind or sea waves have a Gaussian distribution. When we try to record real life stress histories, they usually have also an almost ideal Gaussian characteristic. For the purpose of calculations we assume that the almost ideal signals are Gaussian. It doesn't affect the calculations if we filter the histories correctly (Pawliczek and Kluger 2013). We are dealing with serious issues when we are working with signals that are not nearly Gaussian and we have to calculate the fatigue life. There are some fresh solutions related to that problem presented by Bracessi et al (2009) or Wolfsteiner and Sedlmair $(2013,2015)$. The solutions have the intention to either correct the Gaussian damage degree or to filter the nonGaussian signal into an equivalent Gaussian signal (when it comes to statistical information) with the use of correction factors. We can assess the life interval of a structural component related to its fatigue with the use of either standard stand tests or computation methods. These methods can be divided into two groups defined in the time domain which are based on cycle counting procedures, or those defined in the frequency domain which are using the statistic information of the load. That are so called spectral methods. They use the power spectral density (PSD) to describe the stress amplitudes. The spectral method for fatigue life assessment has many advantages such as the computation time. But the main drawback consists in calculation methods applied in the case of a non-Gaussian random loads. It works perfectly on signals with Gaussian load characteristic, but when we try to calculate non-Gaussian characteristics we often obtain fatigue lives that are overestimated a few times. The paper deals with fatigue life assessment of the 10HNAP steel under nonGaussian loading condition with and without the influence of the mean stress effect. To compensate the mean stress effect a procedure proposed by Niesłony and Böhm $(2012,2015)$ has been used. The non-Gaussianity of a signal has been corrected by the correction factor by Braccesi et al (2009). The fatigue lives estimated on the basis of combination of spectral method and the Dirlik model have been compared with results obtained when the rainflow cycle counting method and experimental data were applied. Damage accumulation for both cases has been calculated with the use of the Palmgren-Miner hypothesis.

\section{FATIGUE STAND TESTS}

The fatigue stand tests have been performed by Łagoda for the 10HNAP steel. Basic material constants can be found in the paper by Łagoda et al. (2001) and are presented in Tab. 1. The samples were tested on the fatigue test stand SHM250 for random tension-compression. A signal has been generated that has a non-Gaussian probability distribution with four dominating frequencies for the case of zero mean stress and non-zero mean stress. The tests with non zero mean stress have been performed for a large influence of the negative stress value. The Probability distribution function of the signal has been presented in Fig. 1. The rainflow matrix of the stress history has been presented in Fig. 2. 
Tab. 1. Mechanical properties of 10HNAP steel

\begin{tabular}{|c|c|c|c|c|c|}
\hline$R_{\boldsymbol{e}}, \mathrm{MPa}$ & $\boldsymbol{R}_{\boldsymbol{m}}, \mathrm{MPa}$ & $\boldsymbol{A}_{10}, \%$ & $\mathbf{Z}, \%$ & $\boldsymbol{E}, \mathrm{GPa}$ & $\boldsymbol{v}$ \\
\hline 414 & 556 & 31 & 35 & 215 & 0.29 \\
\hline
\end{tabular}

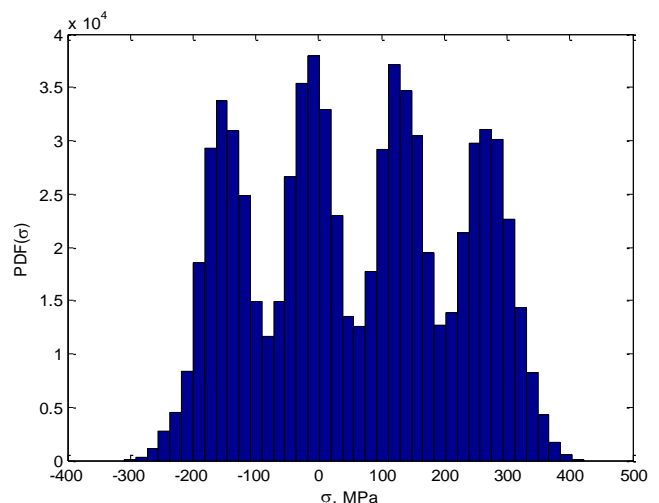

Fig. 1. Probability distribution function (PDF) of the non-Gaussian stress signal

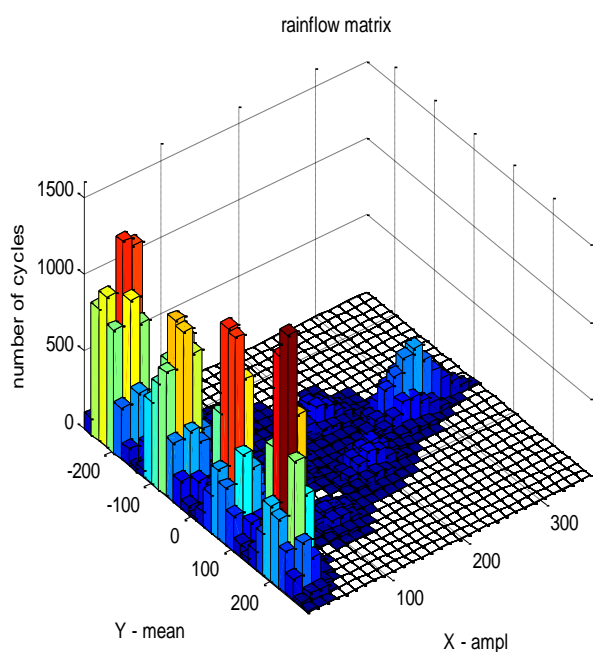

Fig. 2. Rainflow matrix of the stress history

\section{FATIGUE LIFE CALCULATION}

The fatigue life has been calculated with the use of spectral method with the Dirlik model and with the cycle counting method with the use of rainflow cycle counting procedure. The mean stress effect has been compensated with the use of the procedure proposed by Niesłony and Böhm. This one is based on the transformation of a zero mean stress PSD with a correction factor according to the equation:

$G_{\sigma T}(f)=K_{i}^{2}\left(\sigma_{m}\right) \cdot G_{\sigma}(f)$,

where: $G_{\sigma}(f)$ is the PSD function of a centered stress course, $f$ is the frequency, $K_{i}$ is the mean stress correction factor according to a used compensation model, which can be defined exemplary as $K_{G}$ for the Gerber mean stress compensation model as:

$K_{G}=\frac{1}{1-\left(\frac{\sigma_{m}}{R_{m}}\right)^{2}}$,

where: $\sigma_{m}$ - mean value of the stress course, $R_{m}$ - ultimate tensile strength.

The transformed PSD is used to calculate its first five moments $\xi_{k}$ : $\xi_{k}=\int_{0}^{\infty} G_{\sigma T}(f) f^{k} d f$

that are needed to calculate the probability distribution function (PDF). We can find many proposals in the literature that are used for the PDF calculations. These solutions have found their place in certain areas of the industry:

For the marine industry:

Rice (1944) PDF:

$p(\sigma)=\frac{1}{\sqrt{2 \pi \xi_{0}}}\left(1-\gamma^{2}\right)^{\frac{1}{2}} \exp \left[\frac{-\sigma^{2}}{2 \mu^{2}\left(1-\gamma^{2}\right)}\right]+$

$+\frac{\sigma}{2 \mu^{2}}\left[1+\operatorname{erf}\left(\frac{\sigma}{\xi_{0} \sqrt{2 \gamma^{-2}-2}}\right)\right] \exp \left(-\frac{\sigma^{2}}{2 \mu^{2}}\right)$

where: $\mu$ - variance, and $\gamma$ can be calculated with the use of spectral moments $\xi_{i}$ :

$\gamma=\frac{\xi_{2}}{\sqrt{\xi_{0} \xi_{4}}}$

and the function $\operatorname{erf}(u)$ is the error function of stress signal value finding in $[0, u]$ interval:

$\operatorname{erf}(u)=\frac{2}{\sqrt{\pi}} \int_{0}^{u} e^{-t^{2}} d t$.

Chadbury and Dover (1985) have used the Rice formula and proposed the weighted amplitude PDF:

$\sigma_{a w}=\left(2 \sqrt{2 \xi_{0}}\right)\left[\begin{array}{l}\left.\left(\frac{\left(\sqrt{1-\gamma^{2}}\right)^{m+2}}{2 \sqrt{\pi}}\right) \Gamma\left(\frac{m+1}{2}\right)+\frac{\gamma}{2} \Gamma\left(\frac{m+2}{2}\right)+\right]^{\frac{1}{m}} \\ +\operatorname{erf}(\gamma) \frac{\gamma}{2} \Gamma\left(\frac{m+2}{2}\right)\end{array}\right]^{\frac{m}{2}}$

Hancock (1985):

$\sigma_{a w}=\left(2 \sqrt{2 \xi_{0}}\right)\left[\gamma \cdot \Gamma\left(\frac{m}{2}+1\right)\right]^{\frac{1}{m}}$

where $\Gamma$ is the gamma function.

For the aviation industry:

Lallane (2013):

$p(\sigma)=\sqrt{\frac{1-\gamma^{2}}{2 \pi \xi_{0}}} \exp \left(\frac{-\sigma^{2}}{2 \xi_{0}\left(1-\gamma^{2}\right)}\right)+$

$+\frac{\gamma \sigma}{2 \xi_{0}} \exp \left(\frac{-\sigma^{2}}{2 \xi_{0}}\right)\left(1-\operatorname{erf}\left(\frac{\gamma \sigma}{\sqrt{2 \xi_{0}\left(1-\gamma^{2}\right)}}\right)\right)$

Electronic industry:

Steinberg (2000):

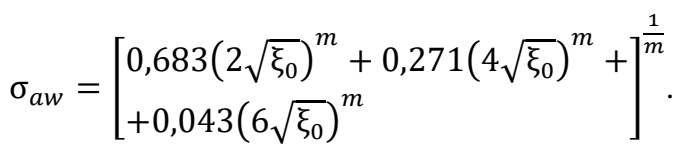

Railway industry:

Tunna (1986)

$p(\sigma)=\frac{\sigma}{4 \gamma \xi_{0}} \exp \left(\frac{-\sigma^{2}}{8 \gamma \xi_{0}}\right)$.

We can also find universal solutions:

By Rayleigh (Bendat 1964):

$p(\sigma)=\frac{\sigma}{\xi_{0}} \exp \left(\frac{-\sigma^{2}}{2 \xi_{0}}\right)$,

and later deviation of Rayleigh's formula by Bendat (1964):

$p(\sigma)=\frac{\sigma}{4 \xi_{0}} \exp \left(\frac{-\sigma^{2}}{8 \xi_{0}}\right)$. 
According to the Dirlik's (1985) distribution function:

$p\left(\sigma_{a}\right)=\frac{1}{2 \sqrt{\xi_{0}}} \cdot\left[\frac{K_{1}}{K_{4}} \cdot e^{\frac{-Z}{K_{4}}}+\frac{K_{2} \cdot Z}{R^{2}} \cdot e^{\frac{-Z^{2}}{2 \cdot R^{2}}}+K_{3} \cdot Z e^{\frac{-Z^{2}}{2}}\right]$,

where: $Z, K_{1}, K_{2}, K_{3}, K_{4}, R$ - factors which are functions of the first five moments of the transformed PSD.

Zhao and Baker (1992):

$p(\sigma)=w \cdot \alpha \cdot \beta \cdot \sigma^{(\beta-1)} \exp \left(-\alpha \sigma^{b}\right)+(1-w) \sigma \exp \left(\frac{-\sigma^{2}}{2}\right)$,

where $w, \alpha, \beta$ are factors described as:

$w=\frac{1-\gamma}{1-\sqrt{\frac{2}{\pi}} \Gamma\left(1+\frac{1}{\beta}\right) \alpha^{\frac{-1}{\beta}}}$,

$\alpha=8-7 \gamma$,

$\beta= \begin{cases}1.1 ; & \gamma<0.9 \\ 1.1+9(\gamma-0,9) ; & \gamma \geq 0,9\end{cases}$

Benassciutti and Tovo (2005) proposed a direct damage function:

$E\left(D_{R F C}\right)=v_{p} C-1 \gamma\left(\sqrt{2 \xi_{0}}\right)^{m} \Gamma\left(1+\frac{m}{2}\right)[b+$

$\left.(1-+b) \gamma^{m-1}\right]$,

where: $C$ and $m$ are constants from the Basquin curve, $b$ is weight function dependant from the PSD, $v p$ is calculated with the formula:

$v_{p}=\frac{1}{2 \pi} \sqrt{\frac{\xi_{4}}{\xi_{2}}}$.

Lutes and Sarkani (1996):

$p(\sigma)=\frac{\sqrt{1-\gamma^{2}}}{\sqrt{2 \pi \xi_{0}}} \exp \left(\frac{\sigma^{2}}{2 \mu^{2}\left(1-\gamma^{2}\right)}\right)+$

$+\frac{\gamma \sigma}{\mu^{2}} \exp \left(\frac{\sigma^{2}}{2 \mu^{2}}\right) \Phi\left(\frac{\gamma \sigma}{\xi_{0} \sqrt{1-\gamma^{2}}}\right)$

A plot of some of these functions has been presented in Fig.3.

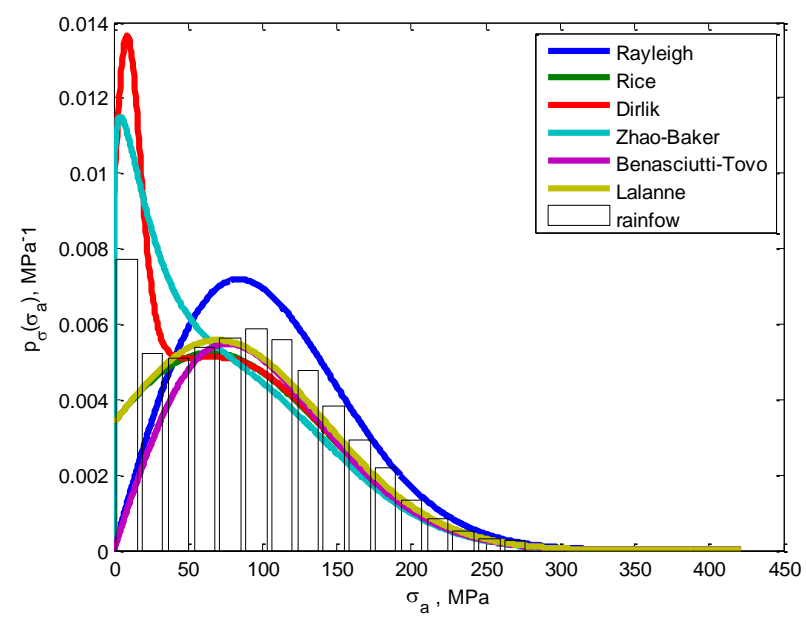

Fig. 3. Chosen probability distribution function shapes for random data in comparison with rainflow amplitude distribution

Fatigue life is calculated using the selected hypotheses of fatigue damage accumulation, e.g. for a linear Palmgren-Miner hypothesis with the amplitude below the fatigue limit we obtain:
$T_{c a l}=\frac{1}{E[P] \int_{0}^{\infty} \frac{p\left(\sigma_{a}\right)}{N\left(\sigma_{a}\right)} d \sigma_{a}} \cdot \frac{1}{\lambda_{n g}}$,

where $E[P]$ is the expected number of peaks, and nonGaussianity is corrected with a corrective factor according to Braccesi et al. (2009):

$\lambda_{n g}=\exp \left(\frac{m^{\frac{2}{3}}}{\pi}\left(\frac{K-3}{5}-\frac{s^{2}}{4}\right)\right)$,

where $m$ - slope of Wöhler curve, $K$ - kurtosis and $S$ - skewness (asymmetry of PDF).

\section{RESULTS AND DISCUSSION}

The calculation of fatigue lifetime has been performed with three calculation methods according to rainflow cycle counting method and with the use of Dirlik's model for spectral method. For the case of non-zero mean stress all methods used the Gerber mean stress compensation model. Calculations according to spectral method have been modified with a corrective coefficient. The obtained power spectrum density has been presented in Fig. 4: The calculations have been compared with experimental results and have been illustrated in a comparison graphs presented in Fig. 5 and Fig. 6

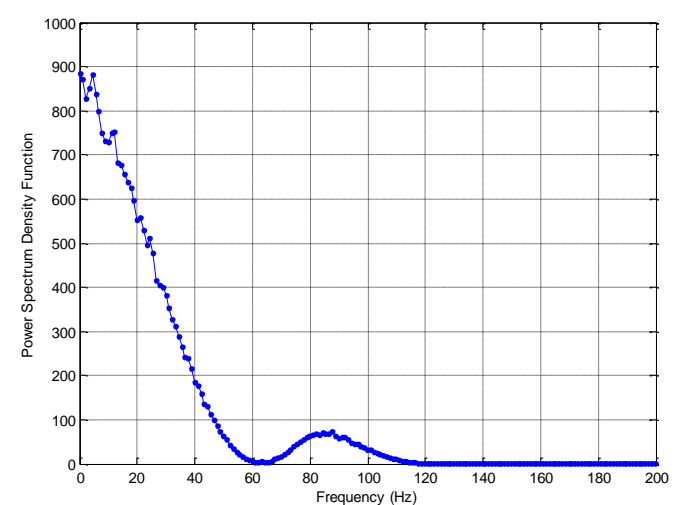

Fig. 4. Course of power spectrum density of the non-Gaussian stress signal versus signal frequency

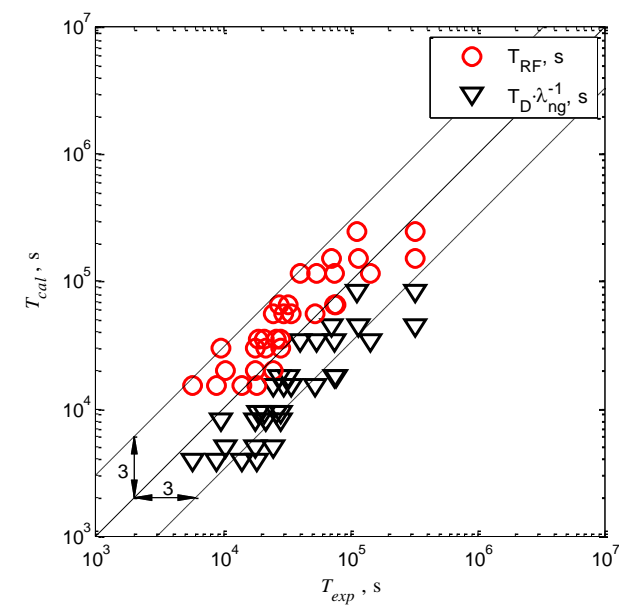

Fig. 5. Comparison of experimentally recorded lifetimes with the calculated results on the basis of rain-flow cycle counting method $T_{R F}$ and the Dirlik model $T_{D}$ for zero-mean stress signal 


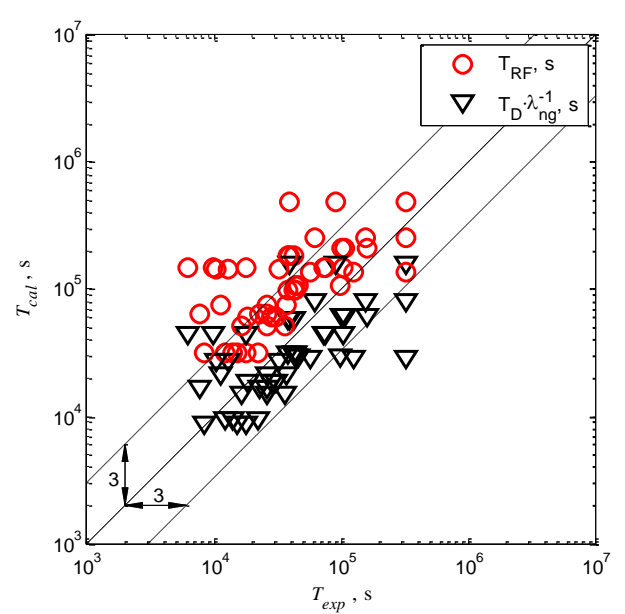

Fig. 6. Comparison of lifetime experimental results with the calculated onces on the basis of rain-flow cycle counting method $T_{R F}$ and the Dirlik model $T_{D}$ with mean stress correction factor

\section{CONCLUSIONS AND OBSERVATIONS}

The fatigue lifetime assessment for the case of non-Gaussian loading histories with four dominating frequencies gives satisfied results after the use of the corrective factor $\lambda_{n g}$. It is possible to use the correction coefficient for such loading cases. It can be awaited that this method will be possible to use for such loading cases. The results indicate that in the case of non-zero mean stress non-Gaussian loading histories the use of spectral method with the correction factor can be applied with the success. Calculations of fatigue lifetime with the use of the Gerber compensation model allow to obtain satisfied results and take into account the impact of compression, when the tests are proceeded with a large influence of negative mean stress value.

\section{REFERENCES}

1. Banvillet A., Łagoda T. , Macha E., Niesłony A., Palin-Luc T., Vittori J.-F. (2004), Fatigue life under non-Gaussian random loading from various Models, International Journal of Fatigue, 26, 349-363.

2. Benasciutti D., Cristofori A.,Tovo R. (2013) Analogies between spectral methods and multiaxial criteria in fatigue damage evaluation Probabilistic Engineering Mechanics, 31, 39-45.

3. Benasciutti D., Tovo R. (2005), Spectral methods for lifetime prediction under wide-band stationary random processes, International Journal of Fatigue, 27 867-877.

4. Benasciutti D., Tovo R. (2006) Comparison of spectral methods for fatigue analysis of broad-band Gaussian random processes, Probabilistic Engineering Mechanics, 21(4), 287-299.

5. Benasciutti D., Tovo R. (2010) On fatigue cycle distribution in nonstationary switching loadings with Markov chain structure, Probabilistic Engineering Mechanics, 25(4), 406-418.

6. Bendat J.S. (1964)., M.A. Corporation, U.S.N.A. and S. Administration, Probability functions for random responses: prediction for peaks, fatigue damage, and catastrophic failures, National Aeronautics and Space Administration.

7. Braccesi C., Cianetti F., Lori G., Pioli D. (2009), The frequency domain approach in virtual fatigue estimation of non-linear systems: The problem of non-Gaussian states of stress, International Journal of Fatigue, 31(4), 766-775.

8. Chaudhury G., Dover W. (1985), Fatigue analysis of offshore platforms subject to sea wave loadings, International Journal of Fatigue, 7, 13-19.

9. Dirlik T. (1985), Application of computers in fatigue analysis, phd thesis University of Warwick.
10. Hancock J., D. Gall (1985), Fatigue under narrow and broad band stationary loading, Marine Technology Directorate Ltd.

11. Lagoda T., Macha E., Pawliczek R. (2001),The influence of the mean stress on fatigue life of 10HNAP steel under random loading, International Journal of Fatigue, 23, 283-291.

12. Lalanne C. (2013), Mechanical Vibration and Shock Analysis, Fatigue Damage, John Wiley \& Sons.

13. Lutes L.D. (1996), Stochastic Analysis of Structural and Mechanical Vibrations, 1st edition, Prentice Hall, Upper Saddle River, N.J.

14. Niesłony A., Böhm M. (2012), Mean Stress Value in Spectral Method for the Determination of Fatigue Life, Acta Mechanica et Automatica, 6 , 71-74.

15. Niesłony A., Böhm M. (2015), Mean Stress Effect Correction in Frequency-domain Methods for Fatigue Life Assessment, Procedia Engineering,12(101), 347-354.

16. Pawliczek R., Kluger K. (2013) Influence of the irregularity coefficient of loading on calculated fatigue life, Journal of Theoretical and Applied Mechanics, 51(4), 791-798.

17. Rice S.O. (1944), Mathematical Analysis of Random Noise, Bell SystemTechnical Journal, 23 282-332.

18. Steinberg D.S. (2000), Vibration Analysis for Electronic Equipment, 3 edition, Wiley-Interscience, New York.

19. Tunna J.M. (1986), Fatigue Life Prediction for Gaussian Random Loads at the Design Stage, Fatigue \& Fracture of Engineering Materials \& Structures, 9, 169-184.

20. Wolfsteiner P., Breuer W. (2013) Fatigue assessment of vibrating rail vehicle bogie components under non-Gaussian random excitations using power spectral densities, Journal of Sound snd Vibration, 332(22), 5867-5882

21. Wolfsteiner P., Sedlmair S. (2015), Deriving Gaussian Fatigue Test Spectra from Measured non Gaussian Service Spectra, Procedia Engineering, 101, 543-551.

22. Zhao W., Baker M. (1992), On the probability density function of rainflow stress range for stationary Gaussian processes, International Journal of Fatigue, 14 (2), 121-135.

Acknowledgements: The Project was financed from a Grant by National Science Centre (Decision No. 2013/09/N/ST8/04332). 